\title{
Ustekinumab Biotherapy and Real-Time Psoriasis Capacitance Mapping: A Pilot Study
}

\author{
Claudine Piérard-Franchimont and Gérald E. Piérard \\ Laboratory of Skin Bioengineering and Imaging, Department of Dermatopathology, University Hospital of Liège, \\ 4000 Liège, Belgium \\ Correspondence should be addressed to Gérald E. Piérard, gerald.pierard@ulg.ac.be
}

Received 10 January 2012; Accepted 1 February 2012

Academic Editor: Enzo Berardesca

Copyright ( 2012 C. Piérard-Franchimont and G. E. Piérard. This is an open access article distributed under the Creative Commons Attribution License, which permits unrestricted use, distribution, and reproduction in any medium, provided the original work is properly cited.

\begin{abstract}
In recent years, the treatment of moderate to severe psoriasis has benefited from the development of targeted biologicals. Assessing this new class of drugs calls for precise modalities of severity/improvement ratings of the disease. Bioengineeringdriven dermometrology aims at improving objective and quantitative assessments of disease severity and treatment efficacy. Skin capacitance mapping/imaging is one of those emerging methods. Among its clinical applications, psoriasis capacitance mapping (PCM) was introduced in order to assess both skin scaliness and water trapping inside the stratum corneum (inflammatory serum deposits) on lesional skin. PCM was used for assessing the therapeutic effects of ustekinumab on target lesions of 5 psoriatic patients. The reduction in the inflammatory dampness of the stratum corneum was conveniently seen after a 1-month ustekinumab treatment. The present pilot study suggests that PCM could be used as a fast and convenient method for assessing the anti-inflammatory efficacy of ustekinumab and other biotherapies.
\end{abstract}

\section{Introduction}

Skin involvement of psoriasis is far from being trivial as it is responsible for significant physical and psychological morbidity. This specific chronic, immuno-inflammatory dermatosis is characterized by the combination of increased keratinocyte proliferation and defective keratinocyte maturation (parakeratosis), associated with angiogenesis and inflammatory cell recruitment [1-3]. The clinicopathological presentations vary according to the type of psoriasis and the body locations [4]. Currently, there is overall agreement in the scientific community to consider psoriasis as an autoimmune disease initiated and mediated by plasmacytoid cells and $\mathrm{T}$ cells $[2,3,5,6]$.

The psoriasis area and severity index (PASI) is considered as an appropriate means for evaluating the global severity of plaque-type psoriasis $[7,8]$. However, in some instances, PASI merely represents a gross evaluation, calling for more precise information regarding the changes in early evolving lesions [9]. Indeed, the interobserver reliability of visual clinical gradings of the global condition is relatively poor
[9-12]. As a result, large variations between estimates of different clinicians impede scoring reproducibility. In addition, the development of active tiny papular lesions of psoriasis is notoriously blurred by the relative extent of the larger plaques [9]. Some objective and quantitative approaches possibly improve the precision and reproducibility of the PASI scoring [11-13]. New assessment methods were further welcomed because recent and upcoming psoriasis treatments offer new opportunities $[6,7,9,14]$, but also fuel some uncertainties regarding the objectivity and specificity of the assessment of psoriasis evolution.

Psoriatic lesions are far less uniform in their histopathological structure than the uninvolved surrounding skin [1]. The density of the inflammatory cell infiltrate, the vascular hyperplasia, the epidermal thickening, and the extent in hyperkeratosis and parakeratosis indeed show large interand intraindividual variations $[1,4,7]$. The regular clinical examination possibly detects some of the variations, but dermoscopy and a few other specific noninvasive bioengineering methods help identifying the clinical heterogeneity with increased specificity and sensitivity $[15,16]$. The same 
methods possibly help assessing the benefits and adverse effects of antipsoriatic therapies, in particular the recent pharmacological advances in biological therapies.

\section{Pathobiology and Functional Changes}

Psoriasis is fostered by the local activation of Th1 and Th17 cells in cooperation with CD123+ plasmacytoid dendritic cells releasing a series of various proinflammatory cytokines $[3,17,18]$.

Among the various cytokines identified in excess in psoriatic lesions [19], TNF- $\alpha$ plays a central role [9]. In addition, the heterodimeric IL-12 and IL-23 cytokines induce naïve $\mathrm{CD} 4+$ lymphocytes to differentiate into Th1 cells and Th17 cells. IL-23 is involved in the regulation of innate defense effector molecules, such as IL-17 and IL-22 present in psoriasis. TNF- $\alpha$ is produced by a wide range of immune and nonimmune cells. It exerts broad inflammatory effects, upregulating both the innate and adaptive immunity. It activates a range of cells, including keratinocytes and dermal dendrocytes. Both IL-12 and IL-23 are produced primarily by plasmacytoid dendritic cells. In turn, the cytokines activate NK cells, CD4+ cells, CD8+ cells, and the differentiation of CD4+ cells into Th1 and Th17 cells. TNF- $\alpha$ triggers the production of IL-1, IL-6, IL-8, and NF- $\kappa$ B contributing to the initiation of a cascade of inflammatory events leading to oedema and recruitment of neutrophils.

\section{Real-Time Psoriasis Capacitance Mapping}

Any change in the structure of the stratum corneum (SC) is potentially associated with alterations of both its barrier function and water holding capacity [20-22]. These changes affect the electrical properties of the skin [23]. In the past decade, a method of nonoptical imaging based on skin capacitance mapping/imaging was developed, showing detailed differences in hydration of the SC over the skin surface [24]. Skin capacitance mapping relies on silicon image sensor technology, initially developed for sensing fingerprints in biometric security procedures [24-27]. Contrasting with skin capacitance mapping, all other available skin electrometric methods only provide average data on SC electrical properties over the area of the measuring probes. With the latter methods, it is impossible to disclose any heterogeneity in skin surface hydration over the test area. In addition, it is impossible to appreciate the relative impact of the partial close apposition of the probe onto rough skin surfaces. This situation obviously interferes with the overage assessment of the global SC hydration.

Psoriasis is conveniently explored using psoriasis capacitance mapping (PCM) which is the application of skin capacitance mapping/imaging to the specific dermatosis [28]. PCM is obtained using the SkinChip device (ST microelectronics, Geneva, Switzerland and L'Oréal, Paris, France). The device is designed for computer recordings of the skin surface hydration and microrelief. The sensor contains 92160 microcapacitors dispersed on a $18 * 12.8 \mathrm{~mm}$ plate protected by a thin silicon oxide layer. It allows simultaneous skin capacitance measurements every $50 \mu \mathrm{m}$.
The probe must be closely applied to the skin surface for about 5 seconds at the most in order to collect relevant information without interference with the water flux and sweat collection inside the SC.

In practice, the real-time skin capacitance nonoptical images are acquired and displayed on a computer screen where capacitance values present as pixels within a range of 256 gray levels. When a close contact is secured between the probe and the skin surface, the darker pixels correspond to high capacitance spots, and the clear pixels reveal spots with lower capacitance values $[24,26,29,30]$. The PCM-derived mean gray level is representative of the average skin surface hydration.

Psoriasis is the paradigm of inflammatory hyperkeratotic dermatoses. PCM reveals a patchwork of different electrical properties on lesional skin [28]. Whitish low capacitance is typical for stable hyperkeratotic plaques. More acute inflammation and evolving plaques show a darker density of high-capacitance spots [28]. This aspect is likely related to sites exhibiting increased transepidermal water loss [31]. PCM can thus provide clues of disease activity in any stage of psoriasis and can be used to monitor therapy over time on a target lesion.

\section{Ustekinumab and PCM}

At present, biologicals approved for psoriasis encompass a few TNF antagonists (adalimumab, efalizumab, etanercept) and ustekinumab, an anti-p40 antibody altering the heterodimeric IL-12 and IL-23 cytokine functions. The present observations were focused on 5 patients aged $48 \pm 6$ years suffering from long-standing plaque type psoriasis admixed with smaller papules dispersed on the trunk and limbs. The patients had been enrolled in an ustekinumab trial. In addition to the regular protocol, the present noninvasive PCM observations were conducted with the understanding and consent of the volunteers. In each of them, PCM assessments were performed on five psoriatic papular lesions located on the trunk and each of the limbs. The observations were performed at entry in the ustekinumab treatment trial and one month after a single $45 \mathrm{mg}$ ustekinumab subcutaneous injection. No other systemic or topical treatment was used in the meantime.

At entry in the study, the psoriatic lesions corresponded to enlarging papules unresponsive to previous systemic treatments. At the PCM examination, they showed a patchwork of darker and lighter spots Figures 1(a) and 1(b). In each volunteer, the relative area $(\%)$ of the darker spots was averaged for the 5 fields. Results were expressed as means and standard deviations (SD) for the relative area. The mean areas were compared by the Student's $t$-test. Results were considered to be significant at the $5 \%$ critical level $(P<0.05)$. All calculations were performed using SAS (version 8.2 for Windows) and S-PLUS (version 6.1) statistical packages.

Both the patterns and the relative areas of darker spots showed large interlesional variations. When pooling all data ( 5 sites) in the 5 patients before and after a 1-month ustekinumab therapy, a global significant difference $(P<0.01)$ was yielded in the mean relative areas of darker spots which were 


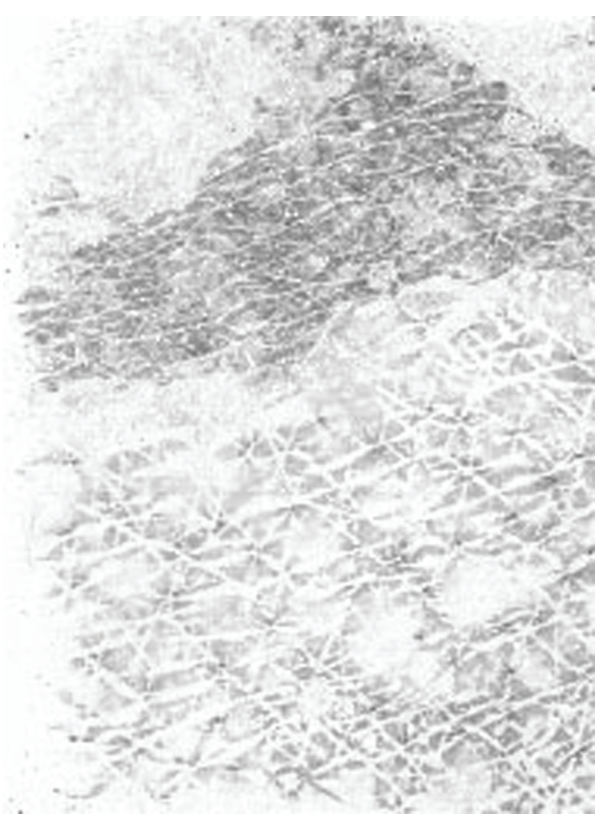

(a) Pretreatment aspect.

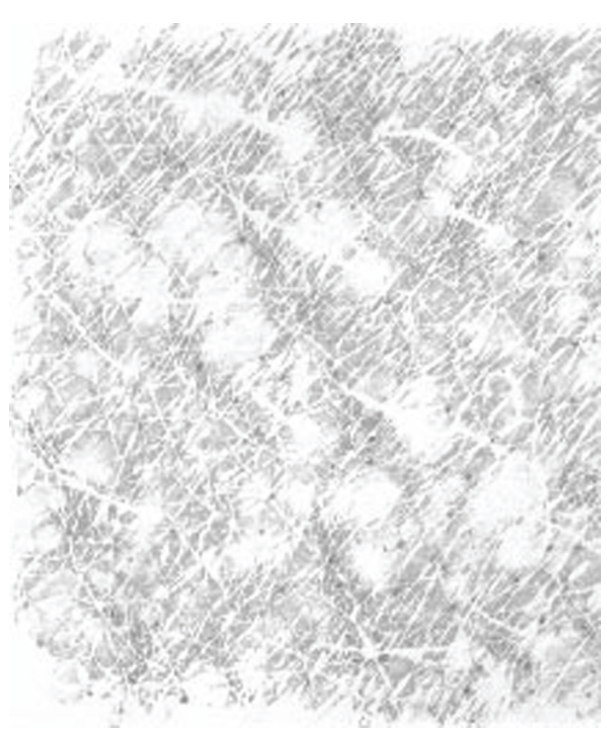

(b) After a 1-month ustekinumab treatment.

FIGURE 1: Skin capacitance mapping of a psoriatic lesion, showing a patchwork of white hyperkeratotic areas and a darker inflammatory area (surface area: $18 * 12.8 \mathrm{~mm}$ ).

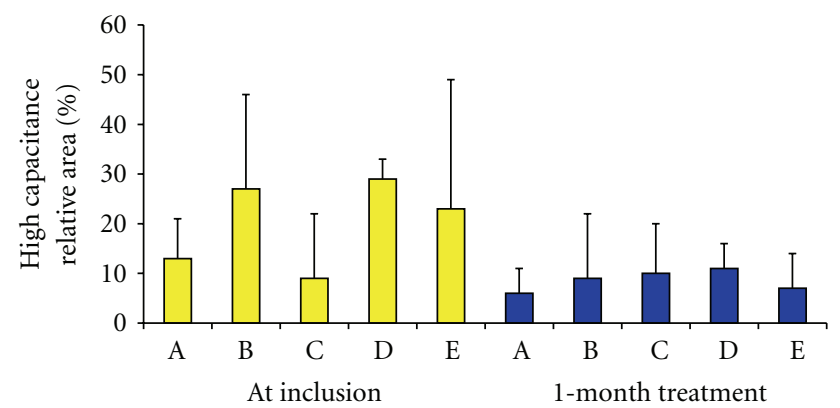

Figure 2: Relative area (means $\pm \mathrm{SD}$ ) of the PCM darker pixels in 5 psoriatic lesions from 5 patients (A to E) at inclusion and after a 1-month ustekinumab treatment. The global reduction in high capacitance inflammatory areas is significant $(P<0.01)$.

abated during the first month of ustekinumab treatment (Figure 2).

\section{Conclusion}

Psoriasis treatment has come a long way for the past decade. In earlier days, the trial-and-error approach to medical innovation represented the roots of psoriasis management. A new era recently emerged when researchers made progress in improving the understanding of the pathobiological processes involved in psoriasis [3, 32]. Tools became available to investigators and clinicians for scrutinizing the biological background supporting the therapeutic efficacy. Research in genetics and immunopathology has improved the understanding of the modalities by which therapies actually work.
It has proved especially rewarding in providing a means for developing appropriate drugs following rational designs [33].

Psoriasis is a chronic, recurrent, immunoinflammatory skin disorder associated with systemic comorbidities [9]. The disease varies in severity and exhibits various morphological subtypes, ranging from a few scaly papules to generalized erythematous plaques and pustules. Depending on the severity of the disease, psoriasis is potentially responsible for major negative physical, economical and psychosocial effects on patients. Recently, specific biologicals were developed to target some steps of the psoriatic biopathology. Ustekinumab is one of the promising drugs in this field [34].

The present pilot study suggests that PCM is sensitive enough to disclose a decrease in inflammation in evolving psoriatic papules while on early ustekinumab treatment. This sign likely represents the inflammatory step initiating psoriasis progression. PCM could be used to the early identification of responders to biologicals. Indeed, the improvement of psoriasis as revealed by PCM was disclosed in this study after a one-month treatment that is much shorter than the 12-week period usually respected for assessing changes in the PASI score. Of note, the mean time to peak serum ustekinumab concentration is about 12 days.

Plaque psoriasis is the most common form, affecting approximately $85-90 \%$ of individuals with the condition. The disease manifests as raised, well-demarcated, erythematous, and frequently pruritic/painful plaques with silvery scales [2]. Approximately 25\% of individuals with psoriasis develop moderate-to-severe disease with widely disseminated lesions [2]. During psoriasis progression, it is not infrequent to see new early papular lesions that are much more active than 
the larger stable plaques [1]. The present study focused on such guttate papular lesions because it was expected that they could represent more sensitive models for evolving psoriasis.

Psoriasis is associated with multiple comorbidities, including psoriatic arthritis, depression, cardiovascular disease, hypertension, obesity, diabetes, metabolic syndrome, and Crohn's disease $[9,35,36]$. It is unknown if breaking the inflammatory evolution of papular psoriasis represents a marker for the evolution of psoriatic comorbidities. The relationship, if any, between PCM data and the evolution of each of the psoriatic comorbidities remains to be settled.

\section{Acknowledgments}

This work was supported by a grant from the "Fonds d'Investissement de la Recherche Scientifique" of the University Hospital of Liège. No other sources of funding were used to assist in the preparation of this paper. The authors have no conflict of interests that is directly relevant to the content of this paper. The authors appreciate the excellent secretarial assistance of Mrs. Ida Leclercq.

\section{References}

[1] I. Uhoda, G. E. Piérard, C. Piérard-Franchimont et al., "Vascularity and fractal dimension of the dermo-epidermal interface in guttate and plaque-type psoriasis," Dermatology, vol. 210, no. 3, pp. 189-193, 2005.

[2] C. E. Griffiths and J. N. Barker, "Pathogenesis and clinical features of psoriasis," The Lancet, vol. 370, no. 9583, pp. 263-271, 2007.

[3] F. O. Nestle, D. H. Kaplan, and J. Barker, "Mechanisms of disease: psoriasis," New England Journal of Medicine, vol. 361, no. 5, pp. 444-509, 2009.

[4] W. H. P. M. Vissers, J. Roelofzen, E. M. G. J. de Jong, P. E. J. van Erp, and P. C. M. van de Kerkhof, "Flexural versus plaque lesions in psoriasis: an immunohistochemical differentiation," European Journal of Dermatology, vol. 15, no. 1, pp. 13-17, 2005.

[5] E. Guttman-Yassky and J. G. Krueger, "Psoriasis: evolution of pathogenic concepts and new therapies through phases of translational research," British Journal of Dermatology, vol. 157, no. 6, pp. 1103-1115, 2007.

[6] M. A. Lowes, A. M. Bowcock, and J. G. Krueger, "Pathogenesis and therapy of psoriasis," Nature, vol. 445, no. 7130, pp. 866873, 2007.

[7] D. Jullien, J. C. Prinz, R. G. B. Langley et al., "T-cell modulation for the treatment of chronic plaque psoriasis with efalizumab (Raptiva): mechanisms of action," Dermatology, vol. 208, no. 4, pp. 297-306, 2004.

[8] J. Schmitt and G. Wozel, "The psoriasis area and severity index is the adequate criterion to define severity in chronic plaquetype psoriasis," Dermatology, vol. 210, no. 3, pp. 194-199, 2005.

[9] G. E. Piérard, C. Piérard-Franchimont, G. Szepetiuk, P. Paquet, and P. Quatresooz, "The therapeutic potential of TNF$\alpha$ antagonists for skin psoriasis comorbidities," Expert Opinion on Biological Therapy, vol. 10, no. 8, pp. 1197-1208, 2010.

[10] R. Marks, S. P. Barton, D. Shuttleworth, and A. Y. Finlay, "Assessment of disease progress in psoriasis," Archives of Dermatology, vol. 125, no. 2, pp. 235-240, 1989.
[11] B. Ramsay and C. M. Lawrence, "Measurement of involved surface area in patients with psoriasis," British Journal of Dermatology, vol. 124, no. 6, pp. 565-570, 1991.

[12] S. Tiling-Grosse and J. Rees, "Assessment of area of involvement in skin disease: a study using schematic figure outlines," British Journal of Dermatology, vol. 128, no. 1, pp. 69-74, 1993.

[13] Y. M. Yune, S. Y. Park, H. S. Oh et al., "Objective assessment of involved surface area in patients with psoriasis," Skin Research and Technology, vol. 9, no. 4, pp. 339-342, 2003.

[14] E. A. Alwawi, E. Krulig, and K. B. Gordon, "Long-term efficacy of biologics in the treatment of psoriasis: what do we really know?" Dermatologic Therapy, vol. 22, no. 5, pp. 431-440, 2009.

[15] M. Stücker, M. Hoffmann, and P. Altmeyer, "Instrumental evaluation of retinoid-induced skin irritation," Skin Research and Technology, vol. 8, no. 2, pp. 133-140, 2002.

[16] F. V ázquez-López, J. A. Manjón-Haces, C. Maldonado-Seral, C. Raya-Aguado, N. Pérez-Oliva, and A. A. Marghoob, "Dermoscopic features of plaque psoriasis and lichen planus: new observations," Dermatology, vol. 207, no. 2, pp. 151-156, 2003.

[17] R. Sabat, S. Philipp, C. Hoflich et al., "Immunopathogenesis of psoriasis," Experimental Dermatology, vol. 16, no. 10, pp. 779798, 2007.

[18] J. Charles, L. Chaperot, D. Salameire et al., "Plasmacytoid dendritic cells and dermatological disorders: focus on their role in autoimmunity and cancer," European Journal of Dermatology, vol. 20, no. 1, pp. 16-23, 2010.

[19] B. J. Nickoloff, H. Xin, F. O. Nestle, and J. Z. Qin, "The cytokine and chemokine network in psoriasis," Clinics in Dermatology, vol. 25, no. 6, pp. 568-573, 2007.

[20] E. Berardesca and H. I. Maibach, "Transepidermal water loss and skin surface hydration in the non invasive assessment of stratum corneum function," Dermatosen in Beruf und Umwelt, vol. 38, no. 2, pp. 50-53, 1990.

[21] C. M. Lee and H. I. Maibach, "Bioengineering analysis of water hydration: an overview," Exogenous Dermatology, vol. 1, no. 6, pp. 269-275, 2002.

[22] J. A. Bouwstra, A. de Graaff, G. S. Gooris, J. Nijsse, J. W. Wiechers, and A. C. van Aelst, "Water distribution and related morphology in human stratum corneum at different hydration levels," Journal of Investigative Dermatology, vol. 120, no. 5, pp. 750-758, 2003.

[23] E. Berardesca, P. Masson, L. Rodrigues et al., "EEMCO guidance for the assessment of stratum corneum hydration: electrical methods," Skin Research and Technology, vol. 3, no. 2, pp. 126-132, 1997.

[24] J. L. Lévêque and B. Querleux, "SkinChip, a new tool for investigating the skin surface in vivo," Skin Research and Technology, vol. 9, no. 4, pp. 343-347, 2003.

[25] G. E. Piérard and J. L. Lévêque, "What is SkinChip? From silicon image sensor technology to SkinChip," Dermatology, vol. 208, no. 4, pp. 291-292, 2004.

[26] D. Batisse, F. Giron, and J. L. Lévêque, "Capacitance imaging of the skin surface," Skin Research and Technology, vol. 12, no. 2, pp. 99-104, 2006.

[27] E. Xhauflaire-Uhoda and G. E. Piérard, "Contrasted skin capacitance imaging of seborrheic keratoses and melanocytic nevi," Dermatology, vol. 212, no. 4, pp. 394-397, 2006.

[28] E. Xhauflaire-Uhoda, C. Piérard-Franchimont, and G. E. Piérard, "Skin capacitance mapping of psoriasis," Journal of the European Academy of Dermatology and Venereology, vol. 20, no. 10, pp. 1261-1265, 2006. 
[29] E. Xhauflaire-Uhoda, P. Paquet, P. Quatresooz, and G. E. Piérard, "Characterization of the skin using capacitance imaging," Expert Review of Dermatology, vol. 5, no. 2, pp. 149-158, 2010.

[30] E. Xhauflaire-Uhoda, G. E. Piérard, and P. Quatresooz, "The skin landscape following nonoptical capacitance imaging," American Journal of Clinical Dermatology, vol. 11, no. 2, pp. 89-94, 2010.

[31] A. T. J. Goon, G. Yosipovitch, Y. H. Chan, and C. L. Goh, "Barrier repair in chronic plaque-type psoriasis," Skin Research and Technology, vol. 10, no. 1, pp. 10-13, 2004.

[32] A. Blauvlet, "New concepts in the pathogenesis and treatment of psoriasis : key roles for IL-23, IL-17A and TGF- $\beta 1$," Expert Review of Dermatology, vol. 2, pp. 69-78, 2007.

[33] A. Clemmensen, M. Spon, L. Skov, C. Zachariae, and R. Gniadecki, "Responses to ustekinumab in the anti-TNF agentnaïve vs. anti-TNF agent-exposed patients with psoriasis vulgaris," Journal of the European Academy of Dermatology and Venereology, vol. 25, no. 9, pp. 1037-1040, 2011.

[34] J. Weber and S. J. Keam, "Ustekinumab," Biodrugs, vol. 23, no. 1, pp. 53-61, 2009.

[35] U. Mrowietz, J. T. Elder, and J. Barker, "The importance of disease associations and concomitant therapy for the longterm management of psoriasis patients," Archives of Dermatological Research, vol. 298, no. 7, pp. 309-319, 2006.

[36] A. B. Kimball, D. Gladman, J. M. Gelfand et al., "National Psoriasis Foundation clinical consensus on psoriasis comorbidities and recommendations for screening," Journal of the American Academy of Dermatology, vol. 58, no. 6, pp. 10311042, 2008. 


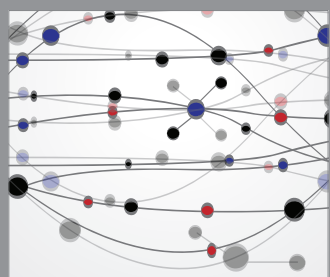

The Scientific World Journal
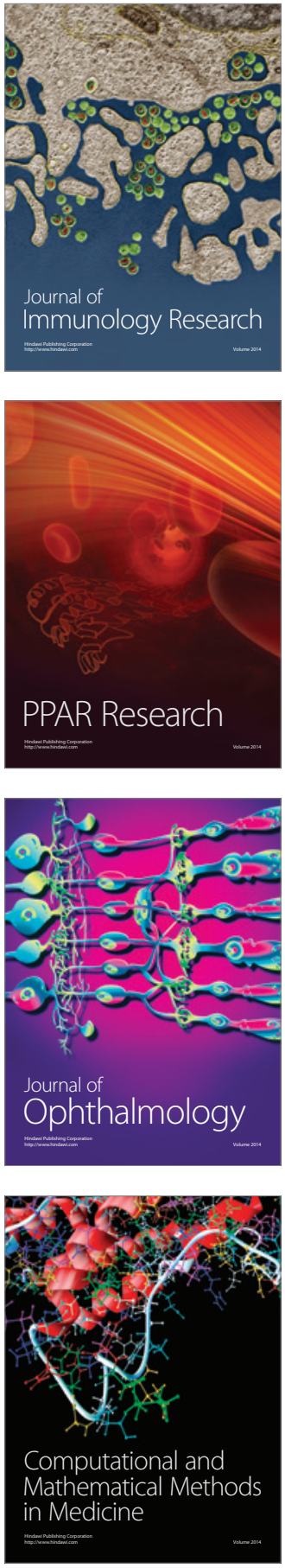

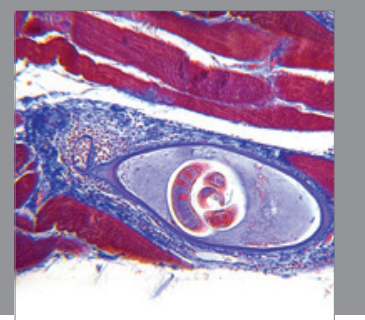

Gastroenterology

Research and Practice
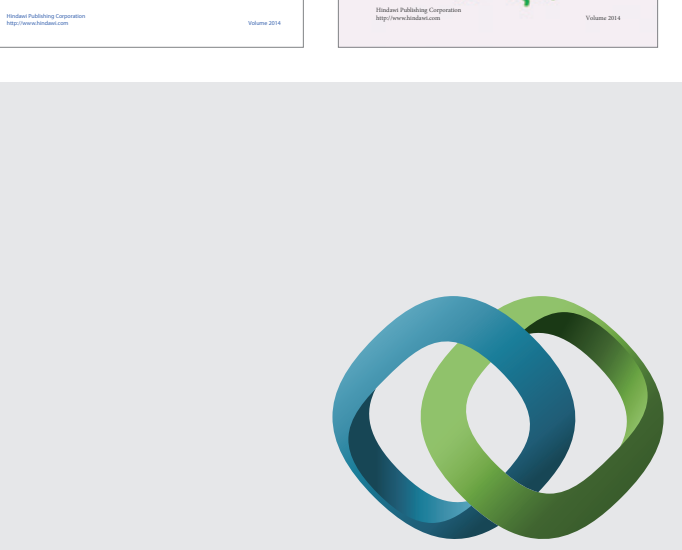

\section{Hindawi}

Submit your manuscripts at

http://www.hindawi.com
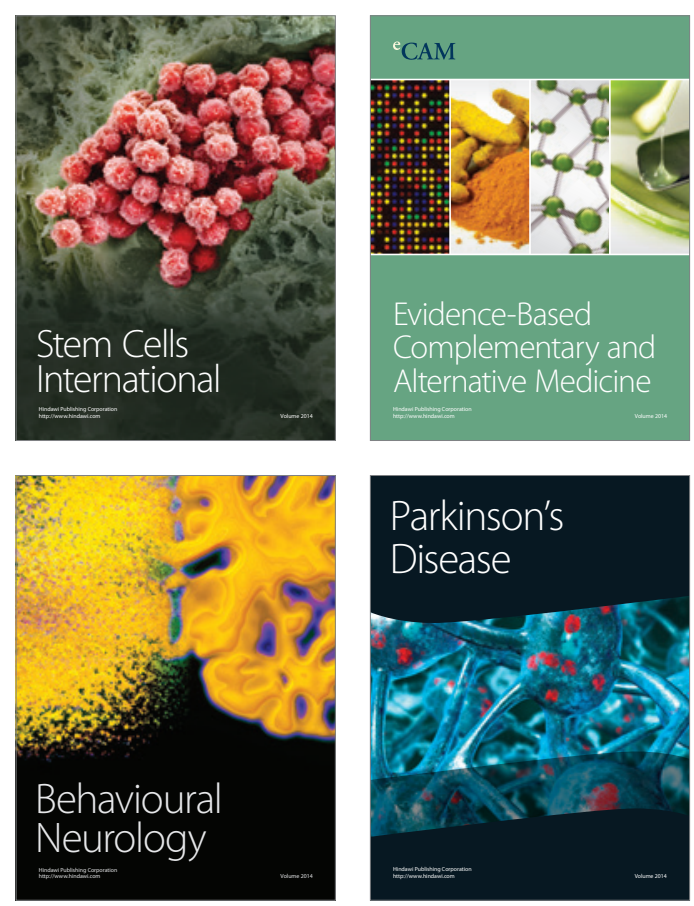

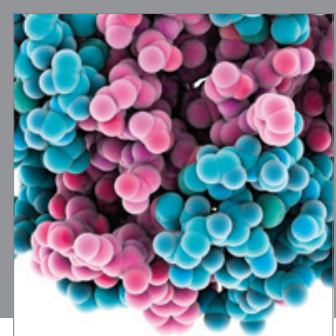

Journal of
Diabetes Research

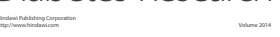

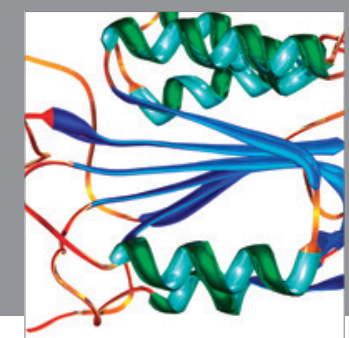

Disease Markers
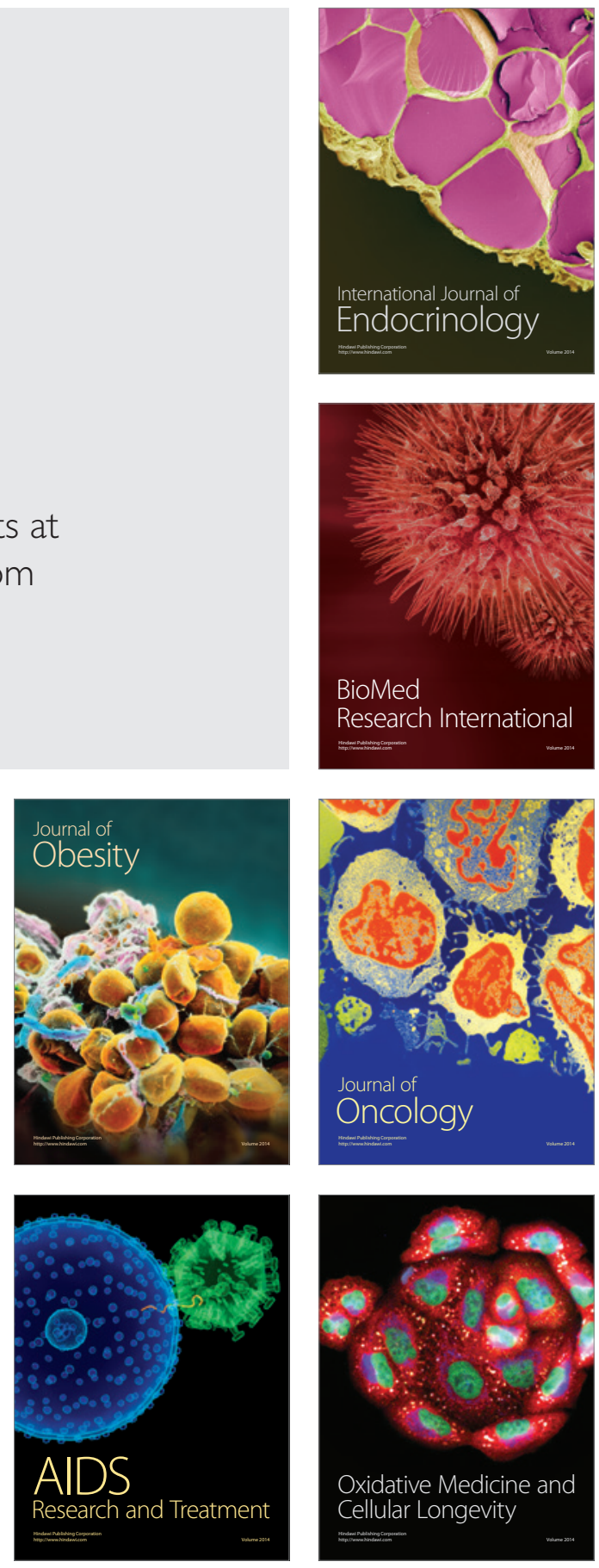